\title{
Leaf Morphology of 89 Tree Species from a Lowland Tropical Rain Forest (Atlantic Forest) in South Brazil
}

\author{
Maria Regina Torres Boeger*, Luiz Carlos Alves and Raquel Rejane Bonatto Negrelle \\ Universidade Federal do Paraná; Setor de Ciências Biológicas; Departamento de Botânica; Laboratório de \\ Ecologia; rboeger@ufpr.br; C.P. 19031; 81.531.990;Curitiba-PR -Brazil
}

\begin{abstract}
We examined the leaf morphology and anatomy of 89 tree species growing in an area of coastal Atlantic Forest in South Brazil. The majority of the species (> 75\%) had small (notophyll and microphyll) elliptical simple leaves with entire margins. These leaves presented a typical anatomical structure consisting of a single epidermal cell layer, single palisade parenchyma cell layer, and spongy parenchyma with 5 to 8 cell layers. The sclerenchyma was limited to the vascular bundles. The majority of the tree species (91\%) had leaves with mesomorphic characteristics. Few species depicted leaves with xeromorphic features as would be expected in such oligotrophic sandy soil. These mesomorphic features appeared to be associated to high efficiency mechanisms for nutrient cycling that compensated for the low nutrient content of the mineral soil.
\end{abstract}

Key words: Leaf morphology; leaf anatomy; lowland tropical forest; Atlantic Forest

\section{INTRODUCTION}

Morpho-anatomical characteristics of the plants are greatly influenced by environmental factors associated with climate, light, amount of rainfall, soil, relief, and altitude (Givnish, 1984). These factors vary in space and time and can be limiting to the establishment and growth of the vegetation. Leaves are directly subjected to these environmental variations and are, thus, important probes for autecological or synecological studies because they evolve specific strategies to certain environmental characteristics (Pyykko, 1979).

The morphology of leaves has been used to describe and compare tropical rain forests (Sobrado and Medina, 1980; Bongers and Popma, 1990; Medina et al., 1990). One of the first authors to use this methodology, Richard (1952), noted that plants of different families in a tropical forest depicted similar morphology while species of the same genera in temperate forests presented distinctive morphological characteristics. Further studies (Grubb et al., 1963; Gentry, 1969) provided support for Richard's hypothesis, showing that $50 \%$ of the species in the lowland tropical forests of Ecuador, Brazil, and Australia presented mesophyllous leaves while $95 \%$ of the plants in montane forests in Costa Rica had microphyllous leaves.

Leaf morphology is also related to environmental gradients (Geeske et al., 1994) and foliar nutrient content (Sobrado and Medina, 1980; Peace and Macdonald, 1981; Körner et al., 1986; Medina et al., 1990). Some tropical plant formations (e.g. campinarana and heath forests) are categorized as sclerophylls based partially on the

*Author for correspondence 
morphology of the leaves of their plant species (Sobrado and Medina, 1980; Turner et al., 1995). Most of these studies, however, have been developed in the tropical forests of the Amazon, Central America, New Zealand, and Hawaii. In the Atlantic Forest, the great majority of the studies are descriptive for a single or for a related group of species (Coutinho, 1962; Souza, 1971; Rôças et al., 1997). The Atlantic Forest extends from $30^{\circ} \mathrm{N}$ to $30^{\circ} \mathrm{S}$ along the coast of Brazil and originally occupied about $1.227 .600 \mathrm{Km}^{2}$. Today, this forest has been reduced to only $7.5 \%$ of its original extent (Myers et al., 2000). It has a high plant diversity (20,000 plant species), among which, 8,000 plant species are endemic (Myers et al., 2000). In the State of Santa Catarina (South Brazil) alone, more than 191 species of trees have been reported (Siqueira, 2001). This study presents the results of a morphological study of 89 tree species from a remnant of the Atlantic Forest located in State of Santa Catarina, Brazil. The morphological patterns reported herein are discussed in relation to environmental conditions and compared to those of other tropical forests.

\section{MATERIAL AND METHODS}

Plant material was collected in the Volta Velha Reserve, a privately owned reserve in South Brazil $\left(26^{\circ} 04^{\prime} \mathrm{S}, 48^{\circ} 38^{\prime} \mathrm{W} \mathrm{Gr}\right)$ and considered part of the Atlantic Forest Biosphere Reserve, located in the Municipality of Itapoá, State of Santa Catarina. The reserve occupies approximately 1.200 hectares and is located in the coastal plain, $5 \mathrm{~km}$ from the Atlantic Ocean and $9 \mathrm{~m}$ above sea level (Fig. 1). The area shows no obvious recent evidence of human disturbance. The forest is classified as a lowland rain forest, with a dense vegetation of 15 to $20 \mathrm{~m}$. tall and is composed by lianas, epiphytes, and palms, besides the tree species (Veloso et al., 1991). The mean annual temperature in the area is $20.3{ }^{\circ} \mathrm{C}$. The hottest month, February, has a mean maximum temperature of $27.2{ }^{\circ} \mathrm{C}$; the coldest month, July, has a mean minimum temperature of $16.5^{\circ} \mathrm{C}$. The mean annual precipitation is $2170 \mathrm{~mm}$. Although there is no distinct dry season, maximum precipitation occurs from January to March.

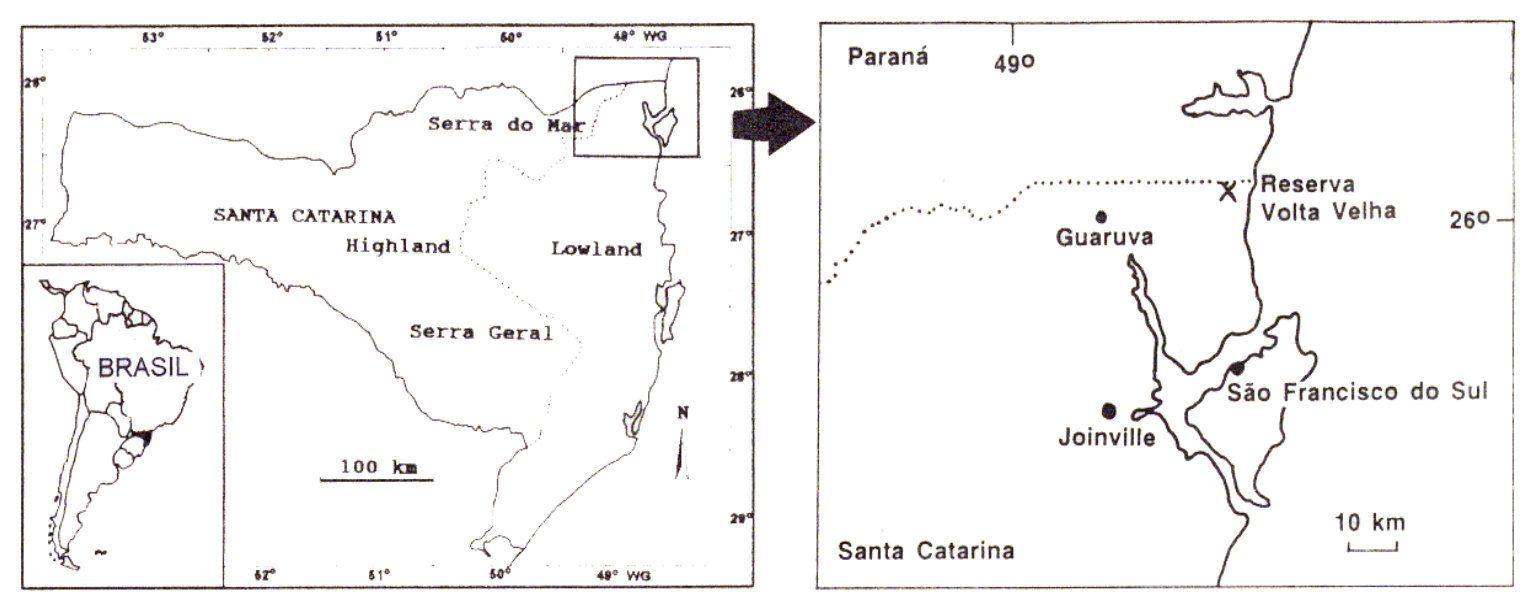

Figure 01 - Location of the study site (Volta Velha Reserve, Santa Catarina State, Brazil).

The soil has been classified as spodsol, nonhydromorphic, with a moderate sand texture, and low concentrations of exchangeable cations (Negrelle, 1995). A previous survey in a 1 ha area found 113 tree species of 34 families. The species studied herein $(n=89)$ were selected based on the number of individuals present in the area (at least 3) and on their high Value of Importance Index (see Negrelle, 1995).

Leaves from branches directly exposed to sunlight (sun leaves) were collected from three individuals of each species. All trees were more than $10 \mathrm{~m}$ tall 
and $10 \mathrm{~cm}$ in diameter at breast height. Leaf area was measured with a leaf-area meter (LiCor, Nebraska, USA) and represented the average values for 20 sun leaves. Classes of leaf size were defined according to Raunkiaer's classification, as modified by Webb (1959). Dried and pressed plant material was also used in the analysis of leaf morphology.

The morphological terminology used was that proposed by Leaf Architecture Working Group (1999). The presence of trichomes and stomata on leaf epidermis was determined using epidermal casts made with colorless nail polish. Sections of $1 \mathrm{~cm}^{2}$ from the central area of each leaf were fixed in 50 FAA (formaldehyde, ethanol $50^{\circ}$, acetic acid
- 18:1:1 v/v), dehydrated with ethanol, embedded in glycol methacrylate resin (Reichert-Jung, Germany), and sectioned at $7 \mu \mathrm{m}$. Sections were stained with toluidine blue (Sass, 1951). All measurements were determined using a light microscope with ocular micrometer. Leaves used in scanning electron microscopy were fixed in 50 FAA, dehydrated through ethanol series to absolute ethanol, and critical-point dried with $\mathrm{CO}_{2}$. Dried specimens were mounted on stubs and coated with gold. Abaxial surfaces were observed with a Philips SEM 505 scanning microscope.

Table 1 - Leaf morphological features of trees analysed from Volta Velha Reserve (SC, Brazil) (n=89).

\begin{tabular}{|c|c|c|c|c|}
\hline Features & & Type: prevalence of & $\operatorname{cies}(\%)$ & \\
\hline Lamina & Simple: 88.5 & Compound: 11.5 & & \\
\hline Leaf symmetry & Symmetrical: 95.0 & Asymmetrical: 5.0 & & \\
\hline Leaf shape & Elliptic: 76.7 & Obovate: 20 & Oblong: 3.3 & \\
\hline Leaf base & Acute: 59.7 & Cuneate: 25.7 & Obtuse: 12.5 & Others: 2 \\
\hline Leaf margin & Entire: 88.8 & Serrate: 7.7 & Erose: 2.4 & Others: 2 \\
\hline Leaf apex & Acuminate: 49.8 & Acute: 30.4 & Obtuse: 8.2 & Others: 11.6 \\
\hline Petiole & Normal: 99 & Absent: 1.0 & & \\
\hline Leaf texture & Coriaceous: 51.7 & Chartaceous: 48.3 & & \\
\hline Trichomes & Present: 40.7 & Absent: 59.3 & & \\
\hline Secretory structures & Present: 41.8 & Absent: 58.2 & & \\
\hline Phyllotaxy & Alternate: 59.1 & Decussate: 21.5 & Opposite: 15.1 & Others: 4.3 \\
\hline $\begin{array}{l}\text { Bundle-sheath } \\
\text { extensions }\end{array}$ & Present: 26.7 & Absent: 73.3 & & \\
\hline Sclerenchyma type & Fibers:72.7 & Sclereides: 3.9 & Absent: 23.4 & \\
\hline $\begin{array}{l}\mathrm{N}^{o} \text { of cell layers in } \\
\text { palisade mesophyll }\end{array}$ & $1: 51.5$ & 2: 29.7 & $\geq 3: 19.8$ & \\
\hline $\begin{array}{l}\mathrm{N}^{\circ} \text { of cell layers in } \\
\text { spongy mesophyll }\end{array}$ & 1 to $4: 15.8$ & 5 to $8: 61.2$ & $\geq 9: 23$ & \\
\hline Leaf size & Notophyllous: 44.1 & Microphyllous: 35.5 & Mesophyllous: 14 & Others: 6.4 \\
\hline
\end{tabular}

\section{RESULTS AND DISCUSSION}

The summary of the morphological analysis of the 89 tree species is presented in Table 1. Main leaf characteristics for all studied species are presented in Table 2. There was no predominance of any state of the following characters: texture, and presence of trichome and secretory structures. Morphologically, the leaves in the area studied (Table 1; Fig. 2) were predominantly simple, symmetrical, elliptic with acuminate apex, entire margin, normal petiole and alternate. These results were very similar to those reported for other tropical forests (Pyykko, 1979; Roth, 1984; Kapelle and Leal, 1996). Elliptical leaves with acuminate or acute apex and entire margins appeared associated with the high pluviosity of the area (Negrelle, 1995).

It has been suggested that this leaf morphology enhances drainage of rainwater, which probably retards the growth of epilhylls and reduces the loss of soluble nutrients by leaching (Whitmore, 1998). The coriaceus texture of leaves observed on more than $50 \%$ of plant species apparently were a defense against herbivory because leaf replacement was an energetically expensive process (Coley and Barone, 1996). Also, the development of this texture has been interpreted as a response to the conditions of water stress and 
high light intensity as that observed in canopies (Roth, 1984).

In the studied area, leaves were predominantly microphyllous and notophyllous ( $>75 \%$ of the species), and smaller than those reported for other lowland tropical forests (Table 3). However, the mesophyll class was overestimated for some tropical forests, because the notophyllous class was also included on the mesophyll class (Webb, 1959; Bongers and Popma, 1990).

In tropical forests, the leaf size was inversely related to the latitude and altitude, and directly related to the annual pluviosity values (Webb, 1959; Dolph and Dilcher, 1980). As precipitation decreased, the average leaf area in a flora also decreased (Webb, 1959; Dolph and Dilcher, 1980). The leaf size was also influenced by others features such as humidity and temperature. Large leaves were more often in warm and moist tropical forests (Werger and Ellenbroek, 1978).

In this study, the predominance of microphyllous and notophyllous leaves appeared to be influenced by the temperature, which was lower at Santa Catarina state when compared to higher temperature of the equatorial regions (Negrelle, 1995).

Crystals occurred in $50 \%$ of the species studied (Table 3). Calcium oxalate crystals are a product of plant metabolism and have been associated with the removal of excess calcium from the system (Volk et al., 2002). The high prevalence of crystals in leaves was also interpreted as a defense against herbivory, by making the leaves less palatable (McKey et al., 1978). According to Aerts and Chapin (2000), leaves with high prevalence of crystals occur mainly in species growing in soils of low fertility where species must avoid loss of leaf tissues and nutrients by the action of herbivores. Only $12 \%$ of the wood species growing in fertile soils (e.g. Los Tuxtlas, Mexico) have crystals in the leaves (Bongers and Popma, 1990).

Table 2 - Main leaf characteristics of studied species from Volta Velha Reserve. Adaxial surface thickness (AdST), abaxial surface thickness (AbST), palisade parenchyma thickness (PPT), spongy parenchyma thickness (SPT), and total thickness (TT).

\begin{tabular}{|c|c|c|c|c|c|c|c|c|c|c|c|c|}
\hline Species & Family & $\begin{array}{c}\text { leaf area } \\
\left(\mathrm{cm}^{2}\right)\end{array}$ & leaf size & lamina & apex & ad. surface & mesophyll type & $\begin{array}{l}\text { AdST } \\
(\mu \mathrm{m})\end{array}$ & $\begin{array}{r}\text { AbST } \\
(\mu \mathrm{m})\end{array}$ & $\begin{array}{l}\text { PPT } \\
(\mu \mathrm{m})\end{array}$ & $\begin{array}{l}\text { SPT } \\
(\mu \mathrm{m})\end{array}$ & $\begin{array}{l}\text { TT } \\
(\mu \mathrm{m})\end{array}$ \\
\hline $\begin{array}{l}\text { Aionea saligna } \\
\text { Alchornea }\end{array}$ & $\begin{array}{l}\text { Lauraceae } \\
\text { Euphorbiaceae }\end{array}$ & 25,74 & notophyll & elliptic & acuminate & uniseriate & asymmetrical & 22,99 & 10,45 & 96,14 & 73,15 & 214,25 \\
\hline $\begin{array}{l}\text { triplinervia } \\
\text { Amaioua }\end{array}$ & Rubiaceae & 46,3 & mesophyll & elliptic & acuminate & uniseriate & asymmetrical & 14,63 & 12,54 & 75,24 & 52,25 & 154,66 \\
\hline $\begin{array}{l}\text { guianensis } \\
\text { Andira }\end{array}$ & Fabaceae & 42,19 & notophyll & elliptic & acuminate & uniseriate & asymmetrical & 20,9 & 10,45 & 48,07 & 96,14 & 175,56 \\
\hline anthelminthica & & 15,81 & microphyll & oblong & acute & uniseriate & homogeneous & 22,99 & 12,54 & 71,06 & 48,07 & 154,66 \\
\hline Aniba firmula & & 36,95 & notophyll & elliptic & acuminate & uniseriate & asymmetrical & 20,9 & 20,9 & 125,4 & 144,21 & 342,8 \\
\hline $\begin{array}{l}\text { Annona cacans } \\
\text { Aparisthmium }\end{array}$ & $\begin{array}{l}\text { Annonaceae } \\
\text { Euphorbiaceae }\end{array}$ & 51,6 & mesophyll & elliptic & acuminate & uniseriate & asymmetrical & 25,08 & 12,54 & 68,97 & 85,69 & 192,28 \\
\hline $\begin{array}{l}\text { cordatum } \\
\text { Byrsonima }\end{array}$ & Malpighiaceae & 143,14 & mesophyll & elliptic & acuminate & uniseriate & asymmetrical & 8,36 & 10,45 & 20,9 & 41,8 & 87,78 \\
\hline $\begin{array}{l}\text { ligustrifolia } \\
\text { Blepharocalyx }\end{array}$ & Myrtaceae & 4,2 & microphyll & elliptic & acuminate & uniseriate & asymmetrical & 31,35 & 20,9 & 73,15 & 152,57 & 277,97 \\
\hline $\begin{array}{l}\text { salicifolius } \\
\text { Cabralea }\end{array}$ & Meliaceae & 11,97 & microphyll & elliptic & acute & uniseriate & symmetrical & 14,63 & 12,54 & 79,42 & 77,33 & 183,92 \\
\hline $\begin{array}{l}\text { canjerana } \\
\text { Calophyllum }\end{array}$ & Clusiaceae & 20,1 & notophyll & elliptic & acuminate & uniseriate & asymmetrical & 16,72 & 12,54 & 35,53 & 87,78 & 152,57 \\
\hline $\begin{array}{l}\text { brasiliense } \\
\text { Calyptranthes }\end{array}$ & Myrtaceae & 30,9 & notophyll & elliptic & obtuse & uniseriate & asymmetrical & 22,99 & 16,72 & 41,8 & 146,3 & 231,39 \\
\hline $\begin{array}{l}\text { concinna } \\
\text { Calyptranthes }\end{array}$ & Myrtaceae & 5,6 & microphyll & elliptic & acute & uniseriate & asymmetrical & 25,08 & 16,72 & 129,58 & 265,67 & 437,07 \\
\hline $\begin{array}{l}\text { lucida } \\
\text { Campomanesia }\end{array}$ & Myrtaceae & 36,27 & notophyll & elliptic & acuminate & uniseriate & asymmetrical & 12,54 & 10,45 & 43,89 & 231,39 & 317,09 \\
\hline guaviroba & & 25,5 & notophyll & elliptic & acuminate & uniseriate & asymmetrical & 18,81 & 8,36 & 66,88 & 83,6 & 179,97 \\
\hline Clethra scabra & C & 31,8 & notophyll & obovate & obtuse & multiseriate & asymmetrical & 14,4 & 48,8 & 100,7 & 119,6 & 287,8 \\
\hline $\begin{array}{l}\text { Clusia criuva } \\
\text { Coccoloba }\end{array}$ & $\begin{array}{l}\text { Clusiaceae } \\
\text { Polygonaceae }\end{array}$ & 25,84 & notophyll & obovate & acute & multiseriate & asymmetrical & 135,8 & 29,6 & 109,9 & 300 & 543,2 \\
\hline $\begin{array}{l}\text { warmingii } \\
\text { Conomorpha }\end{array}$ & Myrsinaceae & 230,91 & macrophyll & elliptic & obtuse & uniseriate & asymmetrical & 35,53 & 14,63 & 77,33 & 52,25 & 163,02 \\
\hline $\begin{array}{l}\text { peruviana } \\
\text { Copaifera }\end{array}$ & Caesalpinaceae & 14,14 & microphyll & elliptic & acuminate & uniseriate & asymmetrical & 25,08 & 14,63 & 48,07 & 121,22 & 214,25 \\
\hline $\begin{array}{l}\text { trapezifolia } \\
\text { Cupania }\end{array}$ & Sapindaceae & 22,06 & notophyll & elliptic & obtuse & uniseriate & asymmetrical & 16,72 & 16,72 & 29,26 & 43,89 & 106,59 \\
\hline oblongifolia & & 92,99 & mesophyll & obovate & obtuse & uniseriate & asymmetrical & 25,08 & 10,45 & 20,9 & 85,69 & 142,12 \\
\hline
\end{tabular}


Cont. Table 2

\begin{tabular}{|c|c|c|c|c|c|c|c|c|c|c|c|c|}
\hline$\overline{\text { Drymys }}$ & Winteraceae & & & & & & & & & & & \\
\hline brasiliensis & & 18,42 & microphyll & obovate & emarginate & uniseriate & asymmetrical & 31,35 & 37,62 & 135,85 & 150,48 & 342,8 \\
\hline Endlicheria & Lauraceae & & & & & & & & & & & \\
\hline paniculata & & 29,94 & notophyll & elíptica & acuminate & uniseriate & asymmetrical & 31,35 & 29,26 & 104,5 & 222,82 & 402,79 \\
\hline $\begin{array}{l}\text { Erythroxylum } \\
\text { vaciniifolium }\end{array}$ & Erythroxylaceae & 3,89 & microphyll & elíntica & retuse & uniser & asvmmetrical & 33.44 & 254 & 3762 & 2958 & 13,18 \\
\hline Esembeckia & Rutaceae & & 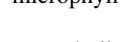 & & & & & & & & 12,000 & \\
\hline grandiflora & & 32,5 & notophyll & elliptic & acute & uniseriate & asymmetrical & 25,08 & 18,81 & 56,43 & 205,68 & 308,52 \\
\hline $\begin{array}{l}\text { Eugenia } \\
\text { cerasiflora }\end{array}$ & Myrtaceae & 40 & notophyll & elliptic & acuminate & uniseriate & asymmetrical & 12,54 & 12,54 & 43,89 & 175,56 & 248,53 \\
\hline Eugenia obovata & Myrtaceae & 17,67 & microphyll & elliptic & acuminate & uniseriate & asymmetrical & 20,9 & 10,45 & 83,6 & 79,42 & 282,81 \\
\hline $\begin{array}{l}\text { Eugenia } \\
\text { subavenia }\end{array}$ & Myrtaceae & 6,3 & microphyll & elliptic & acute & multiseriate & asymmetrical & 48,07 & 20,9 & 117,04 & 274,24 & 454,21 \\
\hline Eugenia tristis & Myrtaceae & 14,07 & microphyll & elliptic & acuminate & uniseriate & homogeneous & 10,45 & 10,45 & 0 & 300,96 & 325,66 \\
\hline Eugenia & Myrtaceae & & & & & & & & & & & \\
\hline umbelliflora & & 35,34 & notophyll & elliptic & acuminate & uniseriate & asymmetrical & 12,54 & 10,45 & 37,62 & 222,82 & 282,81 \\
\hline $\begin{array}{l}\text { Faramea } \\
\text { marginata }\end{array}$ & Rubiaceae & 25,6 & notophyll & elliptic & acuminate & uniseriate & asymmetrical & 14,63 & 6,27 & 39,71 & 96,14 & 156,75 \\
\hline Garcinia & Clusiaceae & & & & & & & & & & & \\
\hline gardneriana & & 27,82 & notophyll & elliptic & acuminate & uniseriate & asymmetrical & 16,72 & 12,54 & 35,53 & 171,38 & 236,17 \\
\hline Gomidesia affinis & Myrtaceae & 17,46 & microphyll & elliptic & acute & unis & asymmetrical & 14,63 & 10,45 & 60,61 & 102,41 & 188,1 \\
\hline Gomidesia & Myrtaceae & & & & & & & & & & & \\
\hline schaueriana & & 25,15 & notophyll & elliptic & acute & multiseriate & asymmetrical & 62,7 & 10,45 & 89,87 & 325,66 & 505,63 \\
\hline $\begin{array}{l}\text { Guarea } \\
\text { macrophylla }\end{array}$ & Meliaceae & & & & & & & & & & & \\
\hline $\begin{array}{l}\text { macrophylla } \\
\text { Guatteria }\end{array}$ & Annonaceae & 29,65 & notophyll & obovate & acuminate & uniseriate & asymmetrical & 25,08 & 16,72 & 64,79 & 158,84 & 65,67 \\
\hline australis & & 15,5 & microphyll & elliptic & acuminate & uniseriate & asymmetrical & 27,17 & 20,9 & 52,25 & 64,79 & 165,11 \\
\hline Heisteria silvianii & $i i$ Olacaceae & 32,65 & notophyll & elliptic & acute & uniseriate & asymmetrical & 22,99 & 14,63 & 41,8 & 169,29 & 274,24 \\
\hline $\begin{array}{l}\text { Hieronyma } \\
\text { alchorneoides }\end{array}$ & Euphorbiaceae & 105,8 & mesophyll & elliptic & obtuse & uniseriate & asymmetrical & 32,8 & 20 & 82,9 & 132,6 & 284,5 \\
\hline Ilex dumosa & Aquifoliaceae & 8,1 & microphyll & elliptic & obtuse & uniseriate & asymmetrical & 22,99 & 18,81 & 137,94 & 119,13 & 299,95 \\
\hline Ilex interregima & Aquifoliaceae & 62,6 & mesophyll & oblong & emarginate & uniseriate & asymmetrical & 37,62 & 25,08 & 299,95 & 257,1 & 634,18 \\
\hline Ilex pseudobuxus & Aquifoliaceae & 5,3 & microphyll & obovate & emarginate & uniseriate & asymmetrical & 20,9 & 10,45 & 125,4 & 68,97 & 267,52 \\
\hline Ilex theezans & Aquifoliaceae & 40,2 & notophyll & obovate & emarginate & uniseriate & asymmetrical & 54,34 & 25,08 & 239,96 & 317,09 & 608,47 \\
\hline Manilkara & Sapotaceae & & & & & & & & & & & \\
\hline subsericea & & 32,3 & notophyll & obovate & acute & multiseriate & asymmetrical & 58,52 & 10,45 & 125,4 & 127,49 & 317,09 \\
\hline Maprounea & Euphorbiaceae & & & & & & & & & & & \\
\hline guianensis & & 6 & microphyll & elliptic & acute & uniseriate & asymmetrical & 22,99 & 22,99 & 52,25 & 77,33 & 173,47 \\
\hline $\begin{array}{l}\text { Marlierea } \\
\text { eugeniopsoides }\end{array}$ & Myrtaceae & 26,76 & notophyll & elliptic & acuminate & uniseriate & asymmetrical & 12,54 & 12,54 & 94,05 & 194,37 & 308,52 \\
\hline Marlierea reitzii & Myrtaceae & 30,2 & notophyll & elliptic & acuminate & uniseriate & asymmetrical & 16,72 & 10,45 & 41,8 & 183,92 & 265,67 \\
\hline $\begin{array}{l}\text { Matayba } \\
\text { guianensis }\end{array}$ & Sapindaceae & 42,5 & notophyll & elliptic & acute & multiseriate & asymmetrical & 35,53 & 25,08 & 60,61 & 81,51 & 202,73 \\
\hline Maytenus robusta & ${ }_{a}$ Celastraceae & 7,6 & microphyll & elliptic & acuminate & uniseriate & asymmetrical & 27,17 & 18,81 & 112,86 & 114,95 & 274,24 \\
\hline Miconia cabuçu & Melastomataceae & 227,04 & macrophyll & elliptic & acuminate & uniseriate & asymmetrical & 22,99 & 10,45 & 33,44 & 106,59 & 173,47 \\
\hline Miconia & Melastomataceae & & & & & & & & & & & \\
\hline $\begin{array}{l}\text { cubatanensis } \\
\text { Miconia }\end{array}$ & & 13,82 & microphyll & elliptic & acute & uniseriate & asymmetrical & 12,54 & 8,36 & 31,35 & 52,25 & 104,5 \\
\hline hymenonervia & & 19 & microphyll & elíptica & acuminate & multiseriate & asymmetrical & 14,63 & 8,36 & 112,86 & 445,64 & 582,76 \\
\hline Miconia & Melastomataceae & & & & & & & & & & & \\
\hline sellowiana & & 20,64 & notophyll & elíptica & acute & uniseriate & asymmetrical & 12,54 & 8,36 & 29,26 & 100,32 & 146,3 \\
\hline Mollinedia & Monimiaceae & & & & & & & & & & & \\
\hline uleana & & 38,36 & notophyll & elliptic & acuminate & uniseriate & asymmetrical & 73,15 & 20,9 & 58,52 & 146,3 & 299,95 \\
\hline Mouriri & Melastomataceae & & & & & & & & & & & \\
\hline chamissoniana & & 40,29 & notophyll & elliptic & acuminate & uniseriate & asymmetrical & 33,44 & 12,54 & 83,6 & 171,4 & 282,81 \\
\hline $\begin{array}{l}\text { Myrceugenia } \\
\text { campestris }\end{array}$ & Myrtaceae & 4.01 & microphyll & obovate & acute & uniseriate & asymmetrical & 25,08 & 10,45 & 104,5 & 202.73 & 342,8 \\
\hline Myrceugenia & Myrtaceae & 4,01 & micropnyi & odovate & acute & uniseriate & asymmetrical & 25,08 & 10,45 & 104,3 & 202,13 & 342,0 \\
\hline reitzii & & 56,1 & mesophyll & elliptic & acute & uniseriate & asymmetrical & 14,63 & 8,36 & 112,86 & 445,64 & 582,76 \\
\hline $\begin{array}{l}\text { Myrcia } \\
\text { acuminatissima }\end{array}$ & Myrtaceae & 14,97 & microphyll & elliptic & acuminate & uniseriate & symmetrical & 12,54 & 6,27 & 35,53 & 85,69 & 140,03 \\
\hline Myrcia fallax & Myrtaceae & 14,14 & microphyll & elliptic & acuminate & uniseriate & symmetrical & 18,81 & 12,54 & 66,88 & 104,5 & 219,45 \\
\hline Nectandra & Lauraceae & & & & & & & & & & & \\
\hline grandiflora & & 20,85 & notophyll & obovate & acuminate & uniseriate & asymmetrical & 27,17 & 14,63 & 100,32 & 135,85 & 277,97 \\
\hline Nectandra & Lauraceae & & & & & & & & & & & \\
\hline megapotamica & & 18,66 & microphyll & elliptic & acuminate & uniseriate & asymmetrical & 25,08 & 10,45 & 89,87 & 108,68 & 231,39 \\
\hline Nectandra & Lauraceae & & & & & & & & & & & \\
\hline oppositifolia & & 72,75 & mesophyll & elliptic & acute & uniseriate & asymmetrical & 13,6 & 23,2 & 103,4 & 64,7 & 210,2 \\
\hline Ocotea aciphylla & Lauraceae & 28,69 & notophyll & elliptic & acute & uniseriate & asymmetrical & 25,08 & 14,63 & 62,7 & 110,77 & 213,18 \\
\hline Ocotea dispersa & Lauraceae & 29,51 & notophyll & elliptic & acuminate & uniseriate & asymmetrical & 20,9 & 16,72 & 5,225 & 125,4 & 231,39 \\
\hline
\end{tabular}




\begin{tabular}{|c|c|c|c|c|c|c|c|c|c|c|c|c|}
\hline Ocotea elegans & Lauraceae & 13,25 & microphyll & elliptic & acuminate & uniseriate & asymmetrical & 16,72 & 12,54 & 35,53 & 140,03 & 325,66 \\
\hline Ocotea glaziovii & Lauraceae & 18,76 & microphyll & elliptic & acuminate & uniseriate & asymmetrical & 25,08 & 12,54 & 83,6 & 114,95 & 231,39 \\
\hline Ocotea odorifera & Lauraceae & 38,08 & notophyll & elliptic & acuminate & uniseriate & asymmetrical & 14,63 & 8,36 & 83,6 & 121,22 & 274,24 \\
\hline Ocotea pulchella & Lauraceae & 7,98 & microphyll & elliptic & acuminate & multiseriate & asymmetrical & 50,16 & 14,63 & 68,97 & 140,03 & 282,81 \\
\hline Ocotea pulchra & Lauraceae & 18,99 & microphyll & elliptic & acuminate & uniseriate & asymmetrical & 22,99 & 14,63 & 75,24 & 114,95 & 222,82 \\
\hline $\begin{array}{l}\text { Oreopanax } \\
\text { capitatum }\end{array}$ & Araliaceae & 51 & mesophyll & obovate & acuminate & multiseriate & homogeneous & 41,8 & 14,63 & 0 & 0 & 222,82 \\
\hline $\begin{array}{l}\text { Ormosia arborea } \\
\text { Ouratea }\end{array}$ & $\begin{array}{l}\text { Fabaceae } \\
\text { Ochnaceae }\end{array}$ & 130,3 & mesophyll & elliptic & acuminate & uniseriate & asymmetrical & 25,08 & 16,72 & 45,98 & 79,42 & 167,2 \\
\hline parviflora & & 14,96 & microphyll & elliptic & acute & uniseriate & asymmetrical & 18,81 & 16,72 & 31,35 & 73,15 & 142,12 \\
\hline $\begin{array}{l}\text { Pera glabrata } \\
\text { Pithecellobium }\end{array}$ & $\begin{array}{l}\text { Euphorbiaceae } \\
\text { Mimosaceae }\end{array}$ & 21 & notophyll & elliptic & acute & uniseriate & asymmetrical & 27,17 & 20,9 & 112,86 & 167,2 & 334,23 \\
\hline langsdorffii & Mimo & 7,1 & microphyll & elliptic & acute & uniseriate & asymmetrical & 16,72 & 16,72 & 73,15 & 71,06 & 177,65 \\
\hline $\begin{array}{l}\text { Pouteria } \\
\text { beaurepairei }\end{array}$ & Sapotaceae & 31,11 & notophyll & obovate & acute & uniestrat & asymmetrical & 25,08 & 20,9 & 29,26 & 112,86 & 188,1 \\
\hline Pouteria venosa & Sapotaceae & 58,91 & mesophyll & obovate & acuminate & uniseriate & asymmetrical & 14,63 & 10,45 & 20,9 & 87,78 & 129,58 \\
\hline Protium kleini & Burseraceae & 19,98 & notophyll & elliptic & acuminate & uniseriate & asymmetrical & 15,2 & 25,6 & 67,9 & 116,4 & 262,8 \\
\hline $\begin{array}{l}\text { Prunus sellowii } \\
\text { Psidium }\end{array}$ & $\begin{array}{l}\text { Rosaceae } \\
\text { Myrtaceae }\end{array}$ & 15,19 & microphyll & elliptic & acute & uniseriate & asymmetrical & 25,08 & 20,9 & 129,58 & 135,85 & 317,09 \\
\hline $\begin{array}{l}\text { catlteyanum } \\
\text { Rapanea }\end{array}$ & Myrsinaceae & 23,14 & notophyll & obovate & acute & multiseriate & asymmetrical & 75,24 & 16,72 & 160,93 & 229,9 & 488,49 \\
\hline ferruginea & & 9,23 & microphyll & elliptic & acute & uniseriate & asymmetrical & 20,9 & 14,63 & 87,78 & 117,04 & 231,39 \\
\hline Rapanea venosa & Myrsinaceae & 29,86 & notophyll & obovate & obtuse & uniseriate & asymmetrical & 41,8 & 35,53 & 68,56 & 222,82 & 359,94 \\
\hline Rudgea villiiflora & Rubiaceae & 33,63 & notophyll & elliptic & acuminate & uniseriate & asymmetrical & 45,98 & 33,44 & 60,61 & 359,94 & 497,06 \\
\hline $\begin{array}{l}\text { Sloanea } \\
\text { guianensis } \\
\text { Solanum }\end{array}$ & $\begin{array}{l}\text { Elaeocarpace } \\
\text { Solanaceae }\end{array}$ & 57,5 & mesophyll & elliptic & acuminate & uniseriate & asymmetrical & 8,36 & 8,36 & 25,08 & 91,96 & 133,76 \\
\hline inaequale & & 21,85 & notophyll & elliptic & acute & úniestrat. & asymmetrical & 14,63 & 14,63 & 20,9 & 48,07 & 98,23 \\
\hline Styrax glabratus & & 33,7 & notophyll & elliptic & acuminate & uniseriate & asymmetrical & 20,9 & 10,45 & 35,53 & 68,97 & 131,67 \\
\hline $\begin{array}{l}\text { Tapirira } \\
\text { guianensis }\end{array}$ & Anacardiaceae & 16,87 & microphyll & elliptic & acuminate & uniseriate & asymmetrical & 27,17 & 18,81 & 167,2 & 114,95 & 299,95 \\
\hline $\begin{array}{l}\text { Ternstroemia } \\
\text { brasiliensis }\end{array}$ & Theaceae & 22,6 & notophyll & obovate & acute & uniseriate & asymmetrical & 23,2 & 40,8 & 184,3 & 333 & 598,2 \\
\hline $\begin{array}{l}\text { Tetrastylidium } \\
\text { grandifolium }\end{array}$ & Olacaceae & 64,8 & mesophyll & elliptic & acute & uniseriate & asymmetrical & 20,9 & 12,54 & 37,62 & 177,65 & 248,53 \\
\hline $\begin{array}{l}\text { Virola oleifera } \\
\text { Weinmannia }\end{array}$ & $\begin{array}{l}\text { Myristicaceae } \\
\text { Cunoniaceae }\end{array}$ & 22,3 & notophyll & oblong & acute & uniseriate & asymmetrical & 27,4 & 19,2 & 87,3 & 100,2 & 249,1 \\
\hline $\begin{array}{l}\text { paulliniifolia } \\
\text { Xylopia }\end{array}$ & Annonaceae & 2,8 & microphyll & obovate & acute & uniseriate & asymmetrical & 29,26 & 14,63 & 158,84 & 121,22 & 325,66 \\
\hline brasiliensis & & 6,12 & microphyll & elliptic & acute & uniseriate & asymmetrical & 18,81 & 14,63 & 91,96 & 64,79 & 190,19 \\
\hline
\end{tabular}

Most species studied (84.9\%) had a single-layered adaxial surface of the epidermis and in all of them, the abaxial surface was formed by a single layer (Table 1, Figs 4c, 4d, 4e, 4f). All species possessed hypostomatic leaves (Figs. 3a, 3b), which could be related to the relative humidity (higher at the abaxial surface than at the adaxial surface) and to temperature (higher at the adaxial surface than abaxial surface) of leaves at the canopy of the forest (Lleras, 1977). Also, the presence of stomata in the abaxial surface could represent a way to prevent the obliteration of the pores of stomata by epyphyllous organisms (Coutinho, 1962).

Although the cuticle of the adaxial surface was not separately measured, most of the investigated species $(75.3 \%)$ had a thick cuticle and several species had an epicuticular wax layer (Figs. 3a, 3b).
Thick cuticle, associated with their chemical composition, helps to repel water, reducing leaching and transpiration (Martin and Juniper, 1970).

Most of the species studied in the Volta Velha Reserve (91\%) had mesomorphic leaves, with a single-layered epidermis, well-developed spongy parenchyma, palisade parenchyma composed of 1 to 2 layers, and reduced supporting tissue (Fig. 4c, $4 \mathrm{~d}, 4 \mathrm{e}, 4 \mathrm{f})$. Only few trees of this area (9\%) presented xeromorphic characteristics such as the presence of sclereids, sub-epidermal layers (Fig. 4a, 4b), bundle-sheath extension (Fig. 4b), and 2 or 3 layers of palisade parenchyma (Fig. 4a; 4b). These features increased the total thickness and also influenced the coriaceous texture of these xeromorphic leaves (Table 4). 


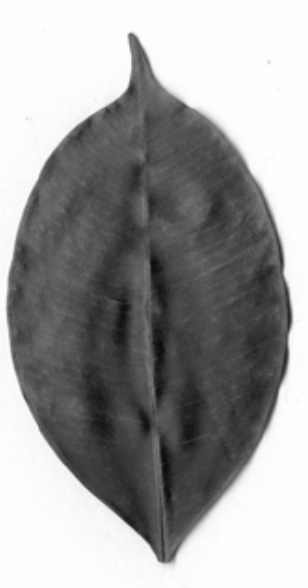

a

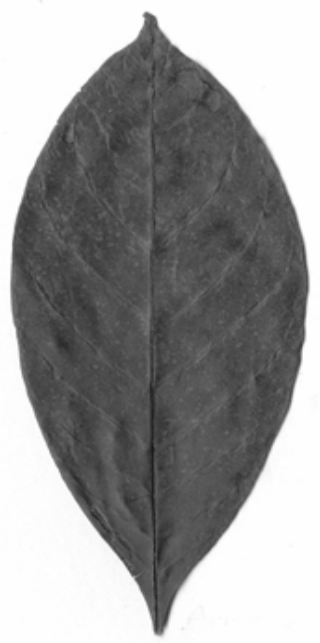

b

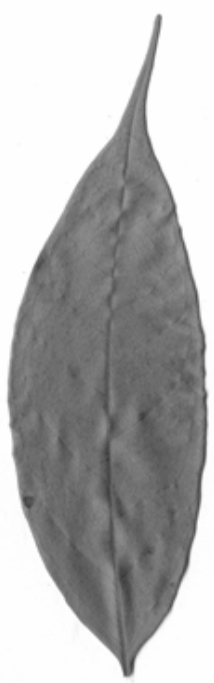

C

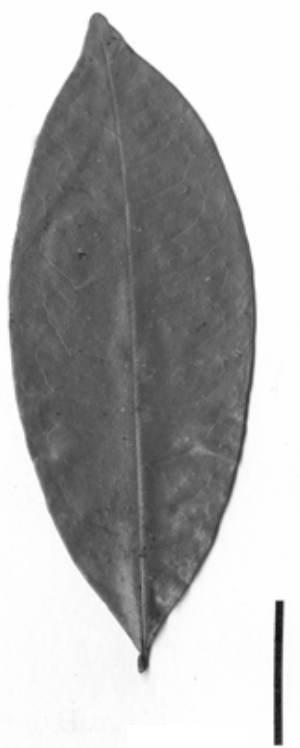

$\mathrm{d}$

Figure 02 - Leaf shapes of tree species from the study area, in the Volta Velha Reserve:

a) Marlierea eugeniopsoides; b) Amaioua guianensis; c) Ocotea aciphylla;

d) Duguetia lanceolata. Scale $=3 \mathrm{~cm}$.

Table 3 - Leaf morphological features (average values) of trees from Volta Velha Reserve (SC - Brazil) and a comparison with other tropical forests.

\begin{tabular}{|c|c|c|c|c|c|c|c|c|c|c|c|c|c|c|}
\hline $\mathbf{A}$ & $\mathbf{B}$ & $\mathrm{C}$ & D & $\mathbf{E}$ & $\mathbf{F}$ & $\mathbf{G}$ & $\mathbf{H}$ & I & $\mathbf{J}$ & $\mathbf{L}$ & $\mathbf{M}$ & $\mathbf{N}$ & $\mathbf{O}$ & $\mathbf{P}$ \\
\hline This study & Brazil & $\begin{array}{l}\text { Lowland } \\
\text { Forest }\end{array}$ & 89 & 58.1 & 269.7 & 78.3 & 145.1 & 0.5 & 10.8 & 26.4 & 16.2 & 44.2 & 40.7 & 100 \\
\hline $\begin{array}{l}\text { Turner et al. } \\
\text { (1995) }\end{array}$ & Malaysia & Heath Forest & 24 & 14.2 & 273 & 96.6 & 112.6 & 0.7 & 28.5 & - & - & - & - & 100 \\
\hline $\begin{array}{l}\text { Medina et al. } \\
(1990)\end{array}$ & Brazil & $\begin{array}{c}\text { Sclerophyllous } \\
\text { Forest }\end{array}$ & 15 & - & 476.4 & $\begin{array}{r}121 . \\
5\end{array}$ & - & - & - & 9.9 & 5.6 & - & - & \\
\hline $\begin{array}{l}\text { Bongers and } \\
\text { Popma (1990) }\end{array}$ & México & $\begin{array}{l}\text { Lowland } \\
\text { Forest }\end{array}$ & 68 & 79.5 & 207.6 & 71.4 & 91.6 & 1.4 & 23 & 14.4 & 30.2 & 12 & 5 & \\
\hline $\begin{array}{l}\text { Tanner and } \\
\text { Kapos (1982) }\end{array}$ & Jamaica & $\begin{array}{c}\text { Upper } \\
\text { Montane }\end{array}$ & 50 & - & 237 & 69.7 & 128.1 & 0.54 & 28 & - & - & 76 & - & \\
\hline $\begin{array}{l}\text { Sobrado and } \\
\text { Medina (1980) }\end{array}$ & Brazil & Bana & 8 & - & 457.4 & - & - & 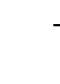 & 62.5 & 17.8 & 10.5 & - & 50 & 100 \\
\hline Pyykko (1979) & Venez. & $\begin{array}{l}\text { Montane } \\
\text { Forest }\end{array}$ & 15 & 86.6 & $80-360$ & - & - & - & 14.3 & - & - & 85.7 & 57.1 & 100 \\
\hline $\begin{array}{l}\text { Grubb et al. } \\
(1975)\end{array}$ & Japan & $\begin{array}{c}\text { Temperate } \\
\text { Rain } \\
\text { Forest } \\
\end{array}$ & 60 & & $0-250$ & - & - & - & 7 & $2-6$ & $1-4$ & 27 & 13 & 98 \\
\hline
\end{tabular}

A: Author; B: Country; C: Forest type; D: Number of species; E: percentage of notophyllous and mesophyllous species; F: Leaf thickness $(\mu \mathrm{m})$; G: Palisade parenchyma thickness $(\mu \mathrm{m})$; H: Spongy parenchyma thickness $(\mu \mathrm{m})$; I: Ratio of spongy/palisade parenchyma; J: Percentage of species with a hypodermis present; L: Adaxial surface thickness ( $\mu \mathrm{m})$; M: Abaxial surface thickness $(\mu \mathrm{m})$; N: Percentage of species with crystals; O: Percentage of species with trichomes; P: Percentage of species with stomata only in the lower epidermis. 

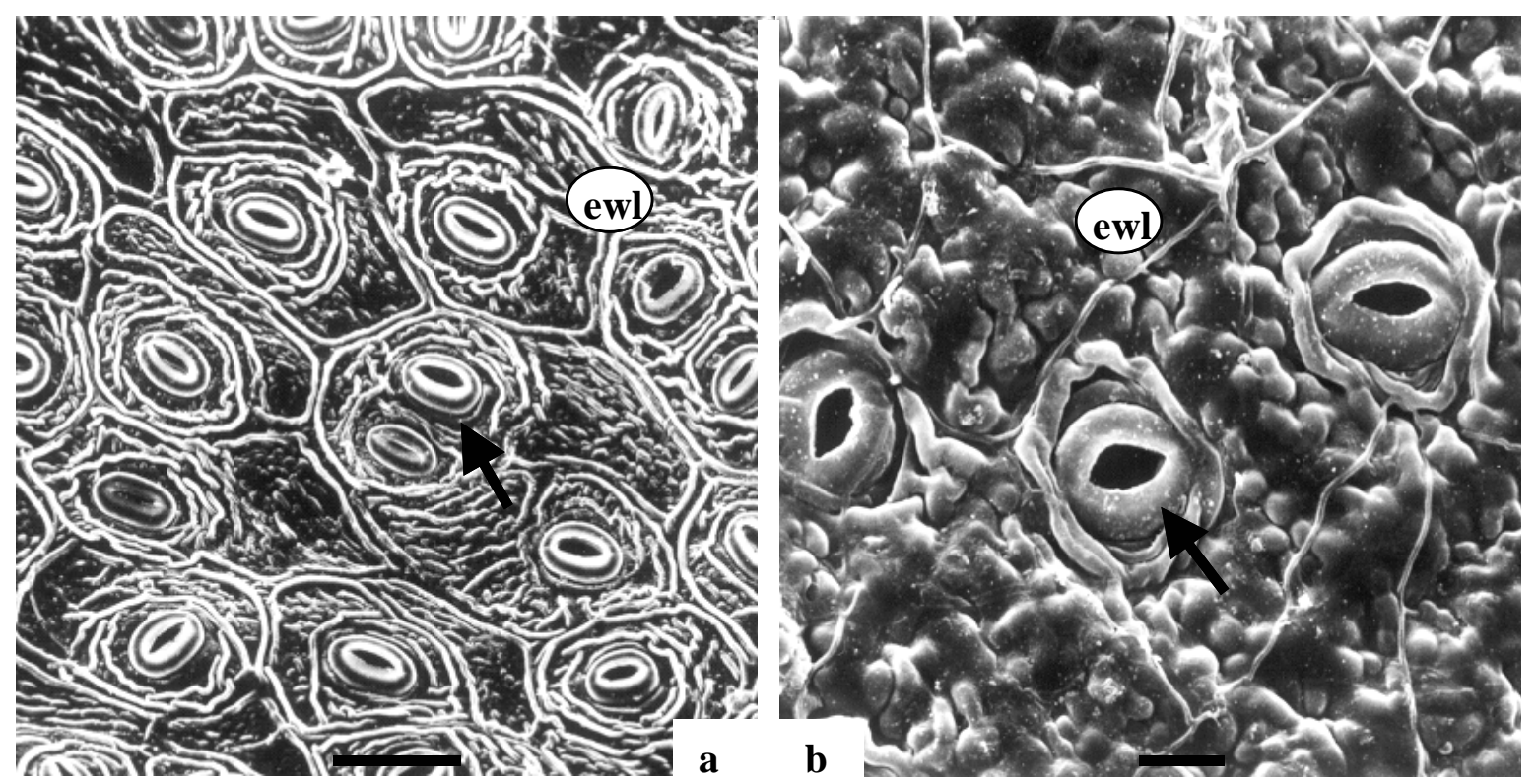

Figure 03 - Stomata (arrow) and epicuticular wax layer (ewl) of the lower epidermis of Rapanea venosa (a) and Clusia criuva (b). Scale $=50 \mu \mathrm{m}$ (a); $10 \mu \mathrm{m}$ (b).

According to Boeger et al. (1997), all species with xeromorphic characteristics are heliophytes occuring at early stages of succession, and have wide geographic distributions (Reitz et al., 1987).

This suggested that the xeromorphic features observed were strategies for survival under adverse environmental conditions, as observed at the early stages of the successional process in sand oligotrophic soils (Boeger et al., 2003).

The morphological parameters of leaves from the most studied species were similar to those of species from other lowland rain forests (Grubb et al., 1975; Pyykko, 1979; Roth, 1984; Bongers and Popma, 1990). This similarity might have resulted from comparable species composition and/or from convergent development as a response to similar environmental conditions (Pyykko, 1979). Most of the studied species (91\%) did not have morpho-anatomical characters (thick leaves, small leaf area, hypodermis, several layers of palisade parenchyma and well developed sclerenchyma) as it would be expected for plants growing on sandy soils of low fertility (see Turner,
1994). The mesomorphic features observed here for most of tree species seem to be related to an efficient nutrient cycling. The litter production in the area was 6.9 ton.ha $^{-1}$.year ${ }^{-1}$ (Boeger et al., 2000) with a slow decomposition rate (data not published). These processes associated with large root biomass and root concentration near surface, in direct contact with the litter (Jordan, 1985) suggested an efficient translocation of foliar nutrients (Vitousek, 1982). These mechanisms expressed by the leaf morphology appear to compensate the low nutrient content of the mineral soil. 

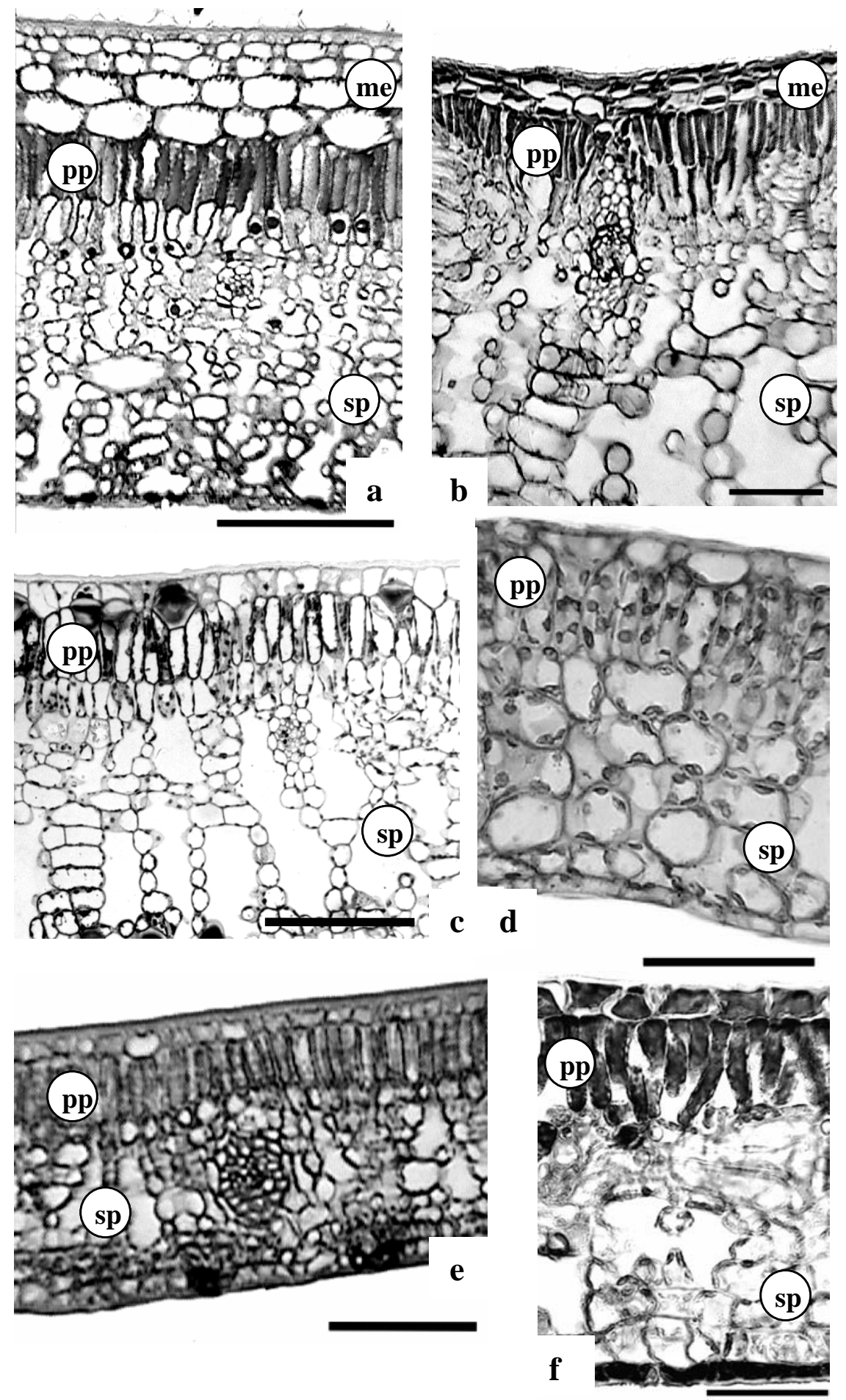

Figure 04 - Transverse sections from the leaf of Clusia criuva (a), Gomidesia schaueriana (b), Ilex theezans (c), Myrcia acuminatissima (d), Pouteria beaurepairei (e), and Tapirira guianensis showing the mesophyll.

(me = multiseriate epidermis; $\mathrm{pp}=$ palisade parenchyma; $\mathrm{sp}=$ spongy parenchyma $)$. Scale $=200 \mu \mathrm{m}$ (a); $100 \mu \mathrm{m}$ (b, c, e); $50 \mu \mathrm{m}$ (d, f). 
Table 4 - Mean values of leaf histological parameters $(\mu \mathrm{m})$ for species with xeromorphic $(\mathrm{n}=8)$ and mesomorphic characteristics $(\mathrm{n}=81)$ and respective standard deviations. * Adaxial surface of the epidermis includes subepidermical layer.

\begin{tabular}{lcc} 
& Xeromorphic plants & Mesomorphic plants \\
\hline Adaxial surface of the epidermis* & $57.7( \pm 36.2)$ & $21.6( \pm 10.8)$ \\
Abaxial surface of the epidermis & $23.2( \pm 9.3)$ & $14.4( \pm 7.3)$ \\
Palisade parenchyma & $166.4( \pm 72.1)$ & $66.4( \pm 48.5)$ \\
Spongy parenchyma & $287.8( \pm 36.7)$ & $121.8( \pm 86.4)$ \\
Total thickness & $533.7( \pm 74.1)$ & $229.0( \pm 23.9)$ \\
\hline
\end{tabular}

\section{ACKNOWLEDGEMENTS}

The authors thank the Machado family, owner of the Volta Velha Reserve; Mr. Jorge Dias, for helping in the fieldwork; the Center of Electronic Microscopy and Laboratory of Structural Botany, UFPR, for allowing use of the equipments; Dr. Delane Kritsky, for reviewing the English version; and FUNPAR, for providing financial support.

\section{RESUMO}

Este estudo examinou a morfologia e anatomia foliar de 89 espécies arbóreas de uma área de Floresta Atlântica Litorânea no Sul do Brasil. A maioria das espécies $(>75 \%)$ possuem folhas pequenas (notófilas e micrófilas) elípticas e simples com margens inteiras. Essas folhas apresentam uma estrutura anatômica formada por epiderme uniseriada, parênquima paliçádico composto por um único estrato de células e o parênquima esponjoso composto por 5 to 8 estratos celulares. O tecido esclerenquimático é limitado aos feixes vasculares. A maioria das espécies arbóreas (91\%) possui folhas com características mesomórficas Poucas espécies (9\%) são xerófilas como era esperado em florestas que crescem sob solo arenoso oligotrófico. As características mesomórficas parecem estar associadas a eficientes mecanismos de ciclagem de nutrientes que compensam o baixo conteúdo de nutrientes do solo mineral.

\section{REFERENCES}

Aerts, R. and Chapin III, F. S. (2000), The Mineral Nutrition of Wild Plants revisited: Re-evaluation of processes and Patterns. Advances in Ecological Research, 30, 1-67.

Boeger M. R. T. and Wisniewski, C. (2003), Comparação da morfologia foliar de espécies arbóreas de três estádios sucessionais distintos de floresta ombrófila densa (Floresta Atlântica) no Sul do Brasil, Revista Brasileira de Botânica, 26, 61-72.

Boeger M. R. T.; Negrelle, R. R. B. and Martins, R. (2000), Produção de serapilheira num gradiente sucessional em floresta ombrófila densa das terras baixas, município de Itapoá (SC). Tecnologia $e$ Ambiente, 6, 91-106.

Boeger, M. R. T.; Brito, C. J. F. A. and Negrelle, R. R B. (1997), Relação entre características morfoanatômicas foliares e esclerofilia em oito especies arboreas de um trecho de floresta pluvial atlantica. Brazilian Archives of Biology and Technology, 40, 493-503.

Bongers F. and Popma, J. (1990), Leaf characteristics of the tropical rain forest flora of Los Tuxtlas, Mexico. Botanical Gazette, 151, 354-365.

Coley, P. D. and Barone, J. A. (1996), Herbivory and plant defenses in tropical forests. Ann. Rev. Ecol. Sys., 27, 305-35.

Coutinho, L. M. (1962), Contribuição ao conhecimento da ecologia da mata pluvial tropical. Boletim da $F$. F.C.L.U.S.P. Botânica, 18, 01-219.

Dolph, G. E. and Dilcher, D. L. (1980), Variation in leaf size with respect to climate in Costa Rica Biotropica, 12, 91-99.

Geeske, J.; Aplet, G. and Vitousek, P. M. (1994). Leaf morphology along environmental gradients in hawaiian Metrosideros polymorpha. Biotropica, 26, 17-22.

Gentry, A. H. (1969), A comparison of some leaf characteristics of tropical dry forest and tropical wet forest in Costa Rica. Turrialba, 19, 419-428.

Givnish, T. J. (1984), Leaf and canopy adaptations in tropical forests. In: Medina, E.; Mooney, H. A. and Vasques-Yanes, C. (Eds.). Physiological ecology of plants in the wet tropics. Dr. W. Junk Publishers, The Hague. pp. 51-84.

Grubb, P. J.; Lloyd, L. R.; Pennington, T. D. and Whitmore, T. C. (1963), Comparison of montane and lowland rain forest in Ecuador. The forest structure, physiognomy, and floristics. J. of Ecology, 5, 567-601. 
Grubb, P. J.; Grubb, E. A. A. and Miyata, I. (1975), Leaf structure and function in evergreen trees and shrubs of Japanese warm temperate rain forest. I. Structure of the lamina. Bot. Mag. Tokyo, 88, 197-211.

Kapelle, M. and Leal, M. E. (1996), Changes in leaf morphology and foliar nutrient status along a successional gradient in a Costa Rican Upper montane Quercus Forest. Biotropica, 28, 331-344.

Körner, C. H.; Bannister, P. and Mark, A. F. (1986), Altitudinal variation in stomatal conductance, nitrogen content and leaf anatomy in different plant life forms in New Zealand. Oecologia, 69, 557-588

Jordan, C. F. (1985), Nutrient cycling in Tropical Forest Ecosystems. Principles and their application in management and conservation. New York : John Wiley \& Sons.

Leaf Architecture Working Group (1999), Manual of Leaf Arquitecture-morphological description and categorization of dicotyledonous and net-veined monocotyledonous angiosperms. Smithsonian Institution. $65 \mathrm{pp}$.

Lleras, E. (1977), Differences in stomatal number per unit area within the same species under different micro-environmental conditions: a working hypothesis. Acta Amazonica, 7, 473-476

Martin, J. T. and Juniper, B. E. (1970), The cuticles of plants. New York : St. Martin's Press.

McKey, D.; Aterman, P. J. W.; Mbi, C. N.; Gartlan, J. S. and Struhsaker, T. T. (1978), Phenolic content of vegetation in two african rain forests: ecological implications. Science, 202, 61-63.

Medina, E.; Garcia, V. and Cuevas, E. (1990), Sclerophylly and oligotrophic environments: relationships between leaf, structure, mineral nutrient content, and drought resistance in tropical rain forest of the upper Rio Negro region. Biotropica, 22, 51-64.

Myers, N.; Mittermeier, R. A.; Mittermeier, C. G.; Fonseca, G. A. B. and Kent, J. (2000), Biodiversity hotspots for conservation priorities. Nature, 403, 853-858.

Negrelle, R. R. B. (1995), Composição florística, estrutura fitossociológica e dinâmica de regeneração da Floresta Atlântica na Reserva de Volta Velha, Município de Itapoá, SC. PhD Thesis. São Carlos, S.P., Brasil.

Peace, W. J. H. and Macdonald, F. D. (1981), An investigation of the leaf anatomy, foliar mineral levels, and water relations of tress of a Sarawak Forest. Biotropica, 13, 100-109.

Pyykko, M. (1979), Morphology and anatomy of leaves from some woody plants in a humid tropical forest of Venezuelan Guayana. Acta Bot. Fennica, 112, 1-41.

Reitz, R.; Klein, R. M. and Reis, A. (1987), Projeto madeira de Santa Catarina. Sellowia, 28. [Suplemento].

Richards, P. W. (1952), The tropical rain forest: an ecological study. Cambridge : University Press.

Rôças, G.; Barros, C. F. and Scarano, F. R. (1997), Leaf anatomy plasticity of Alchornea triplinervia
(Euphorbiaceae) under distinct light regimes in a Brazilian montane Atlantic rain forest. Trees, 11, 469-473.

Roth, I. (1984), Stratification of tropical forest as seen in leaf structure. Tasks for Vegetation Science. Ed. H. Lieth. Junk, The Hague.

Sass, J. E. (1951), Botanical Microtechnique. 2. ed. Iowa State College Press.

Siqueira, M. F. (2001), Listagem de espécies arbóreas do Estado de Santa Catarina. Available http://www.bdt.org.br/mata.atlantica/flora/local?SC.

Sobrado, M. A and Medina, E. (1980), General morphology, anatomical structure, and nutrient content of sclerophyllous leaves of the "bana" vegetation of Amazonas. Oecologia, 45, 341-345.

Souza, A.V. G. (1971), Contribuição ao estudo da anatomia foliar e da fisiologia de Psidium guayava Raddi. Ciência e Cultura, 23,373-382.

Tanner, E. V. J. A. and Kapos, V. (1982), Leaf structure of jamaican montane rain-forest trees. Biotropica, 14, 16-24.

Turner, I. M. (1994), A quantitative analysis of leaf form in woody plants from the world's major brodleaved forest types. Journal of Biogeography, 21, 413-419.

Turner, I. M.; Ong, B. L. and Tan, H. T. W. (1995), Vegetation analysis, leaf structure and nutrient status of a Malaysian heath community. Biotropica, 27, 2-12.

Veloso, H. P.; Rangel, L. R. Filho. and Lima, J. C. A. (1991), Classificação da vegetação brasileira, adaptada a um sistema universal. Rio de Janeiro : IBGE.

Vitousek, P. M. (1982) Nutrient cycling and nutrient use efficiency. The American Naturalist, 119, 553-576.

Volk, G. M.; Lynch-Holm, V. J.; Kostman, T. A.; Goss, L. J. and Franceschi, V. R. (2001), The role of druse and raphide calcium oxalate crystals in tissue calcium regulation in Pistia stratiotes leaves. Plant Biol., 4, 34-45.

Webb, L. J. (1959), A physiognomic classification of australian rain forests. $J$. of Ecology, 47, 551-570.

Werger, M. J. A. and Ellenbroek, G. A. (1978). Leaf size and leaf consistence of a riverline forest formation along a climatic gradient. Oecologia, 34, 297-308.

Whitmore, T. C. (1998), An introduction to tropical rain forests. Oxford : Oxford University Press.

Received: March 24, 2003; Revised: November 18, 2003; Accepted: July 07, 2004. 